\title{
Electron trapping in shear Alfvén waves that power the aurora
}

\section{Article}

Published Version

Watt, C. E. J. and Rankin, R. (2009) Electron trapping in shear Alfvén waves that power the aurora. Physical Review Letters, 102. 045002. ISSN 0031-9007 doi:

https://doi.org/10.1103/PhysRevLett.102.045002 Available at https://centaur.reading.ac.uk/32818/

It is advisable to refer to the publisher's version if you intend to cite from the work. See Guidance on citing.

Published version at: http://dx.doi.org/10.1103/PhysRevLett.102.045002

To link to this article DOI: http://dx.doi.org/10.1103/PhysRevLett.102.045002

Publisher: American Physical Society

All outputs in CentAUR are protected by Intellectual Property Rights law, including copyright law. Copyright and IPR is retained by the creators or other copyright holders. Terms and conditions for use of this material are defined in the End User Agreement.

\section{www.reading.ac.uk/centaur}

\section{CentAUR}

Central Archive at the University of Reading

Reading's research outputs online 


\title{
Electron Trapping in Shear Alfvén Waves that Power the Aurora
}

\author{
Clare E. J. Watt* and Robert Rankin \\ University of Alberta, Edmonton, Alberta, Canada \\ (Received 7 November 2008; published 26 January 2009)
}

\begin{abstract}
Results from 1D Vlasov drift-kinetic plasma simulations reveal how and where auroral electrons are accelerated along Earth's geomagnetic field. In the warm plasma sheet, electrons become trapped in shear Alfvén waves, preventing immediate wave damping. As waves move to regions with larger $v_{T e} / v_{A}$, their parallel electric field decreases, and the trapped electrons escape their influence. The resulting electron distribution functions compare favorably with in situ observations, demonstrating for the first time a selfconsistent link between Alfvén waves and electrons that form aurora.
\end{abstract}

Terrestrial auroral displays may be categorized into two different classes: discrete and diffuse. Discrete aurora consists of well-defined luminous features that are produced by the magnetic field-aligned acceleration of electrons in the magnetosphere above the auroral ionosphere. The nature of this acceleration process can also be divided into different types according to the underlying physics [1]: acceleration through quasistatic field-aligned electric fields which form in a region $(2-3) R_{E}$ radial distance from the Earth above the auroral ionosphere, and acceleration by the transient parallel electric field carried by shear Alfvén waves of small perpendicular extent [2,3]. Shear Alfvén waves in Earth's magnetosphere are guided from the plasma sheet to the ionosphere by the geomagnetic field, and spacecraft observations have demonstrated that they have sufficient energy flux to power terrestrial auroral displays [4]. Observations from satellites also suggest that these dispersive-scale Alfvén waves are coincident with accelerated field-aligned electrons [5]. However, the details of the wave acceleration process are not well understood, in part because the interaction is governed by kinetic processes that are difficult to study without resorting to numerical experiment, and the fact that the waves travel through variable plasma environments between the plasma sheet and the ionosphere. In this Letter, we present results from a simulation of the kinetic interaction between shear Alfvén waves in a nonuniform magnetic field and demonstrate how and where electron acceleration can take place in the Earth's auroral magnetosphere and other inhomogeneous plasma environments.

The plasma sheet supports a warm, tenuous plasma, with electron temperature $T_{e} \sim 100-2000 \mathrm{eV}$, and electron number density $n_{e} \sim 10^{5}-10^{6} \mathrm{~m}^{-3}$. In this region, the electron thermal speed $v_{T e}$ is larger than the local Alfvén speed $v_{A}$, and therefore significant wave-particle interactions are expected when shear Alfvén waves travel through the plasma [6]. Closer to the ionosphere, the magnetic field strength is much larger due to greater proximity to the Earth. In this region, the plasma has $v_{T e} \ll v_{A}$ and the characteristics of the shear Alfvén wave change. In the vicinity of the plasma sheet, the predicted parallel electric field strength is high and the phase velocity of the wave $v_{\mathrm{ph}}>v_{A}$ for short perpendicular wavelengths [7]. In plasma closer to the ionosphere, $v_{\mathrm{ph}}<v_{A}$, and for the same short perpendicular scales, the predicted parallel electric field is reduced [8]. Previous modeling results have demonstrated that the flux and energy of electrons accelerated by shear Alfvén waves depend sensitively on the phase velocity and amplitude of the parallel electric field supported by the wave [9]. It is demonstrated here that in inhomogeneous plasma, the variation of the ratio $v_{T e} / v_{A}$ along the geomagnetic field determines the altitude range of wave-particle interactions which form the aurora.

We present results from DK-1D [8], a nonlinear and selfconsistent drift-kinetic plasma simulation code which has been developed to study the interaction between shear Alfvén waves and electrons in the nonuniform plasma environment of Earth's magnetosphere. Figure 1(a) shows a representation of the simulation domain in near-Earth space. The dashed lines represent an idealized dipolar geomagnetic field, and the thick dark line represents the one-dimensional simulation domain, stretching from around $2.5 R_{E}$ radial distance to nearly $8 R_{E}$ radial distance. The initial electron number density $n_{e}=10^{6} \mathrm{~m}^{-3}$ and electron temperature $T_{e}=200 \mathrm{eV}$ are constant, to elucidate the physics of a shear Alfvén wave traveling along the nonuniform geomagnetic field. Because of the dipolar variation of the magnetic field, the Alfvén speed varies from $\sim 2 \times 10^{6} \mathrm{~m} / \mathrm{s}$ at the upper end of the simulation domain to $\sim 7 \times 10^{7} \mathrm{~m} / \mathrm{s}$ at the lower end of the domain. The perpendicular wave number $k_{\perp}=2 \pi / \lambda_{\perp}$ is scaled along the geomagnetic field according to dipolar mapping [6], given $\lambda_{\perp}=4 \mathrm{~km}$ at a reference altitude of $110 \mathrm{~km}$. Figure 1(b) shows the variation of the perpendicular wave number as a function of distance in the simulation domain, and Fig. 1(c) shows the variation of the ratio $v_{T e}^{2} / v_{A}^{2}$, demonstrating that the simulation covers parameter regimes which are appropriate for the auroral zone magnetosphere. The simulation follows the evolution of three parameters in time on a fixed grid in phase space and 

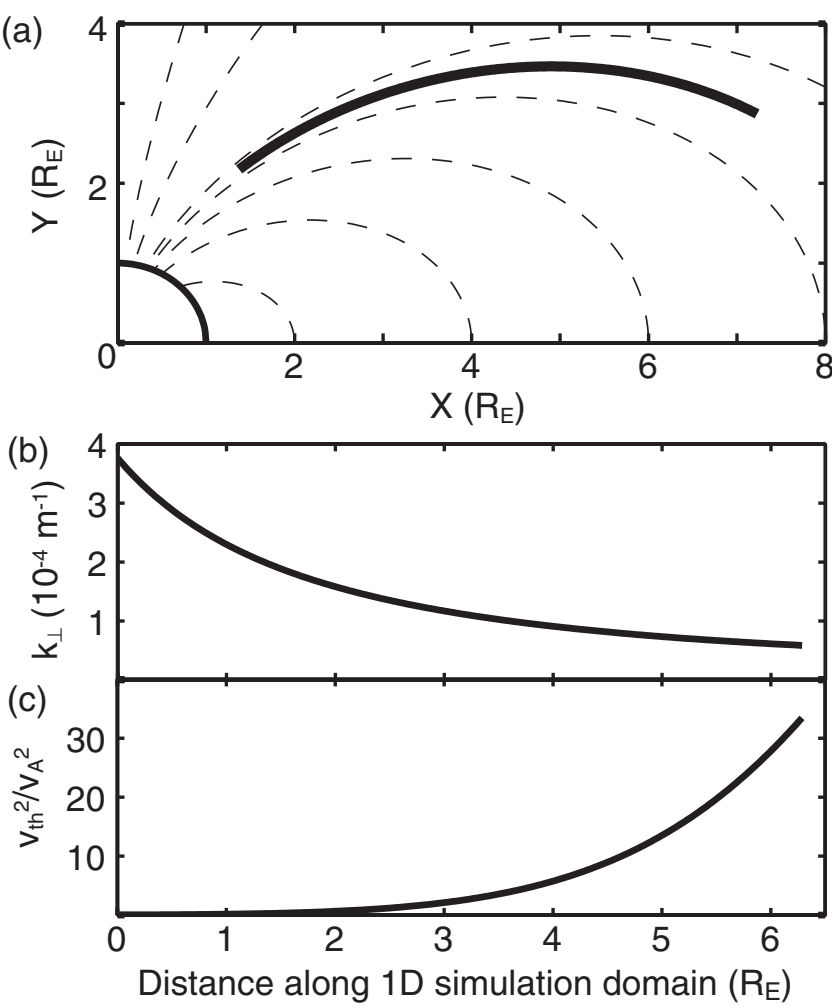

FIG. 1. (a) Graphical representation of the simulation domain in an idealized dipolar magnetosphere, $X$ is the radial distance in the equatorial plane of a dipolar field, and $Y$ is the distance along the magnetic dipolar axis, with the origin at the center of the Earth. (b) Variation of the perpendicular wave number along the simulation domain. (c) Variation of the ratio $v_{T e}^{2} / v_{A}^{2}$ along the simulation domain. The "top" of the simulation is to the right of (b) and (c).

configuration space: the electron distribution function $f_{e}\left(z, p_{\|}, \mu\right)$, the scalar potential $\phi(z)$, and the parallel component of the vector potential $A_{\|}(z)$, where $z$ is the field-aligned coordinate $(z=0$ at the end of the simulation domain closest to the Earth), $p_{\|}=v_{\|}-\left(q_{e} / m_{e}\right) A_{\|}$is the parallel canonical momentum per unit mass, $v_{\|}$is the parallel velocity, and $\mu$ is the magnetic moment, which is assumed to remain constant for each electron.

Figure 2 shows snapshots of $f_{e}\left(z, v_{\|}\right)$for $\mu=0$ (top row) and the parallel electric field $E_{\|}(z)=-\partial A_{\|} / \partial t-$ $\partial \phi / \partial z$ (bottom row) during a simulation which was initialized by adding a wave packet of the form $\phi_{w}=$ $\Phi_{0} \exp \left[-(t-3 T)^{2} / 4 T^{2}\right] \sin (\omega t)$ to the scalar potential at the upper boundary, where the amplitude $\Phi_{0}=100 \mathrm{~V}$, the period $T=2 \mathrm{~s}$, and the frequency $\omega=2 \pi / T$. Linear theory [6] predicts that resonant wave-particle interactions will occur in a region around the phase velocity $v_{\mathrm{ph}} \sim$ $-2 \times 10^{6} \mathrm{~m} / \mathrm{s}$ at the top of the simulation, and that the resonant velocity will increase in magnitude as the wave moves through decreasing $z$. The distribution function snapshots in the upper panels of Fig. 2 indicate that sig- nificant phase space modification is occurring at the predicted resonant velocities for large values of $z$, near the top of the simulation domain. As the wave packet enters the simulation domain, regions of positive $E_{\|}$accelerate electrons downwards. Those electrons which are resonant with the wave (at the wave phase velocity $v_{\mathrm{ph}}<v_{T e}$ ) become preferentially accelerated to form a beam. However, once this beam encounters the region of negative $E_{\|}$immediately ahead, particle trapping occurs, and the wave damping in the warm plasma is momentarily halted.

At locations above $z \sim 4 R_{E}$ in Fig. 2, and for all times indicated, the parallel electric field is near its maximal value, and the distribution function shows distinct trapping regions. Conversely, in the plasma environment below $z \sim$ $4 R_{E}, E_{\|}$is much weaker, and electrons are able to stream down the field unimpeded as the trapping islands lose their shape. The dependence of trapping on $v_{T e} / v_{A}$ is confirmed by simulations with uniform magnetic field and otherwise identical conditions, which reveal that the trapping of electrons persists all along the simulation domain when $B_{0}$ is constant and $v_{T e} / v_{A} \gg 1$. In the homogeneous case, after the initial wave-particle energy exchange at the upper boundary (which creates the trapped populations), the wave amplitude remains essentially constant for all $z$. This confirms that in inhomogeneous plasma, electrons are able to escape the wave and participate in wave acceleration only after they have moved into a different $v_{T e} / v_{A}$ regime. For shear Alfvén waves traveling through warm plasma, $E_{\|} / E_{\perp} \sim k_{\|} k_{\perp} \lambda_{e}^{2} v_{T e}^{2} / v_{A}^{2}$, where $k_{\|}$is the parallel wave number and $\lambda_{e}$ is the electron skin depth [10]. We quantify the changes in the parallel electric field strength in the simulation by noting that the local Alfvén speed changes with $z$, as does the perpendicular scale size. Figure 1(c) shows that the value of $v_{T e}^{2} / v_{A}^{2}$ is very large towards the top of the simulation domain where trapping occurs, and drops off sharply as the wave propagates into regions of increasing magnetic field strength (although $v_{T e}$ is still larger than $v_{A}$ at the altitude at which the electrons begin to escape the influence of the wave). The perpendicular wave number, on the other hand, increases only slightly over the upper part of the simulation domain. Therefore, as the shear Alfvén wave progresses down the simulation domain towards the ionosphere, the parallel electric field strength diminishes due to decreasing $v_{T e}^{2} / v_{A}^{2}$ and the trapped electrons eventually reach an altitude where they may escape the influence of the wave.

The simulation results discussed above suggest a mechanism whereby shear Alfvén waves can propagate through warm plasma at large radial distance from the Earth without experiencing the strong damping that is predicted by linear theory [6]. This is relevant to observations from the NASA Polar spacecraft in the auroral zone [11], which suggest that field-aligned shear Alfvén wave Poynting flux is large beyond a geocentric distance of $4 R_{E}$ and small inside this region, implying that shear Alfvén waves asso- 

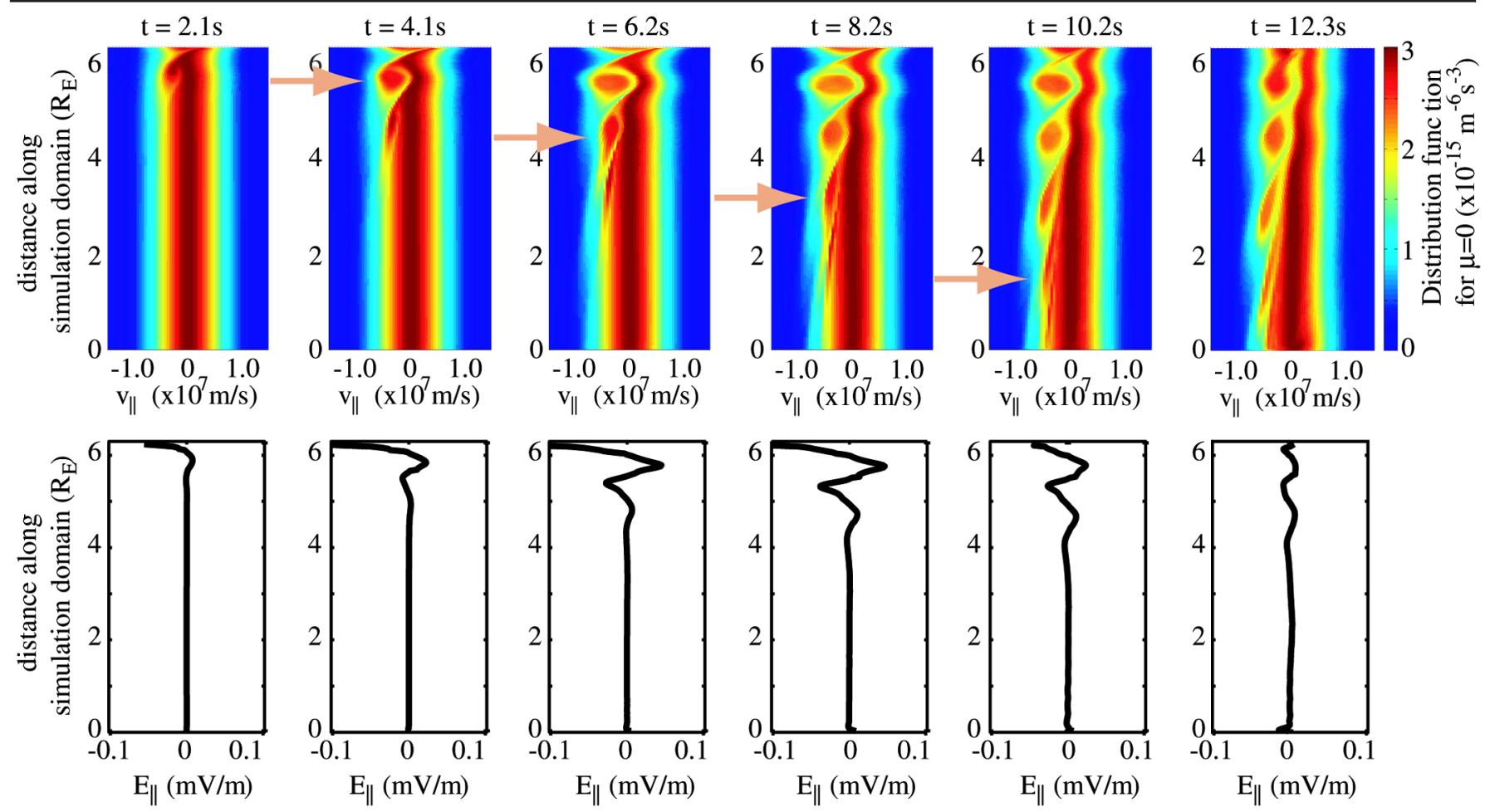

FIG. 2 (color online). Simulation snapshots of the electron distribution function (for $\mu=0$ ) as a function of $z$ and $v_{\|}$(top row) and the parallel electric field as a function of $z$ (bottom row). Orange arrows track a particular phase feature of the wave as it moves through the simulation domain.

ciated with this Poynting flux are losing a significant fraction of their energy at $4 R_{E}$. The nonlinear trapping demonstrated in the simulation results discussed above can explain this observation: wave-particle interactions sustain the shear Alfvén wave beyond a particular radial distance where the parallel electric field carried by the shear Alfvén wave is large. Then, when the wave $E_{\|}$ decreases due to changes in the ambient plasma and magnetic field conditions, the electrons can escape the wave, and provide a sink for the wave energy.

The electron distribution functions that accompany shear Alfvén waves of small perpendicular extent often indicate both parallel and antiparallel energization [12]. To compare our simulation results with these observations from the Polar satellite, we average $f_{e}$ at $z=5.5 R_{E}$ in the simulation domain over an interval of $13 \mathrm{~s}$ to simulate the accumulation time of the particle detector on Polar. Figure 3(a) shows the resulting two-dimensional distribution function for a simulation where the initial pulse amplitude is $\Phi_{0}=100 \mathrm{~V}$. This corresponds to an initial wave Poynting vector with a relatively modest amplitude $\sim 7 \mu \mathrm{W} \mathrm{m}^{-2}$ (when compared with a statistical survey of shear Alfvén wave Poynting vectors in this region of space [4]). Figure 3(a) shows elongation of the distribution function in the parallel direction, which is a signature of both resonant electron acceleration to form the trapped beam as well as acceleration of the bulk distribution function in the opposite direction to carry the parallel current of the shear
Alfvén wave. The details of the distribution function motion can be seen clearly in the upper panels of Fig. 2, where the distribution function is shifted in the upward direction in order to carry the shear Alfvén wave current, whereas the resonant electron beam is accelerated in the downwards direction (the direction of propagation of the wave). Figure 3(b) shows a similarly averaged distribution function from $z=5.5 R_{E}$ for a simulation where the initial wave amplitude was $\Phi_{0}=400 \mathrm{~V}$. The elongation in the parallel velocity direction becomes more pronounced as the wave amplitude is increased, in general agreement with the process observed by the Polar satellite.

Previous simulation studies of wave-particle interactions in shear Alfvén waves [13] have demonstrated that the bulk distribution motion is local: electrons are accelerated and decelerated locally to carry the wave parallel current when required. The trapped electrons observed in the results presented here experience long-range transport from where they are first accelerated near the top of the simulation domain, through the trapping region, where they are confined by successive potential wells in the wave packet, to the free-streaming region below $z \sim 4 R_{E}$, where the most field-aligned electrons now have access to the ionosphere and can contribute towards auroral brightening. Our simulations also reveal that electrons with large values of $\mu$ are reflected above the ionosphere by the mirror force acting in the converging magnetic field, and do not contribute to the auroral brightening seen at lower altitude. 

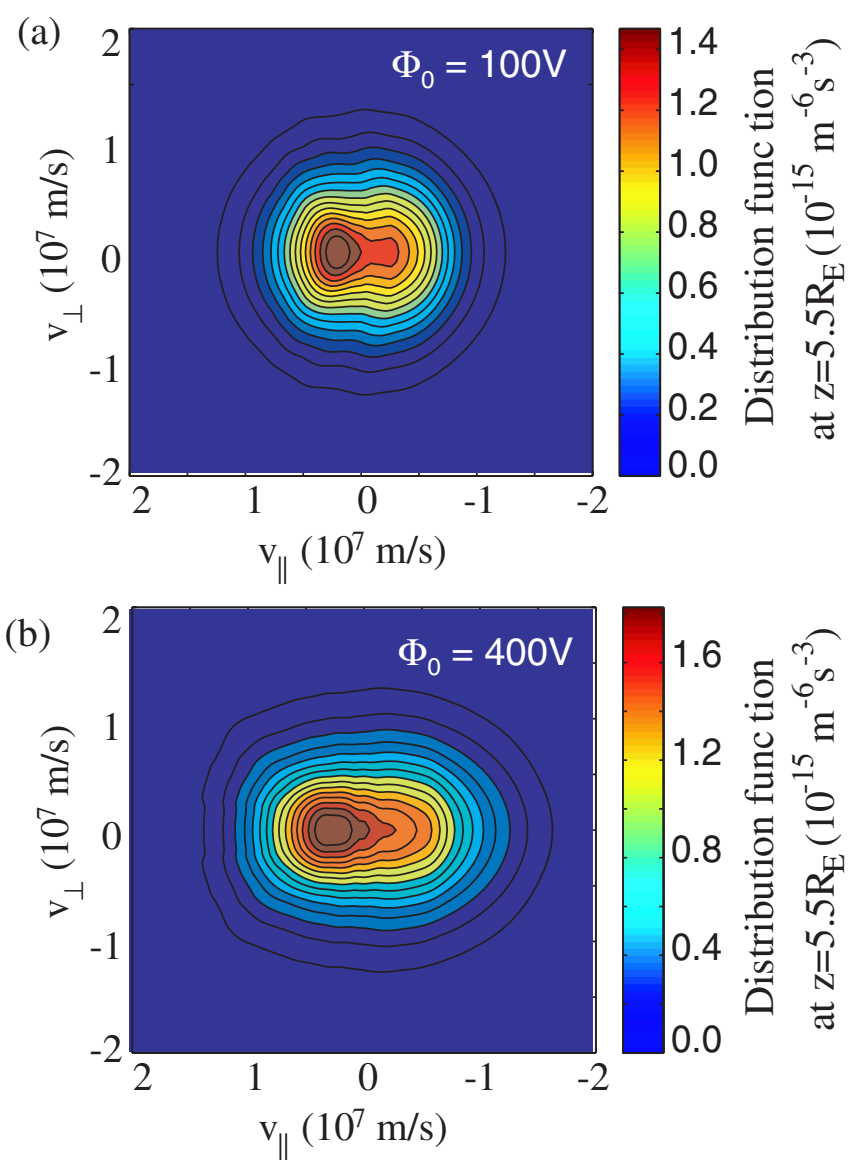

FIG. 3 (color online). Averaged simulation distribution function from $z=5.5 R_{E}$ as a function of parallel and perpendicular velocity for initial wave amplitude of (a) $100 \mathrm{~V}$ and (b) $400 \mathrm{~V}$. Note that the positive and negative directions of the parallel velocity have been reversed for easier comparison with Polar observations [12].

In conclusion, the simulation results shown in this Letter reveal that the nonlinear plasma interaction between shear Alfvén waves and electrons can explain observational findings from the magnetospheric auroral zone. In plasma with $v_{T e}>v_{A}$ (i.e., conditions prevalent in the magnetospheric plasma sheet and other natural plasma environments), nonlinear particle trapping allows shear Alfvén waves to persist in plasma regions where one would otherwise expect large damping. As the waves move into re- gions where different plasma conditions prevail, their parallel electric field is weakened, and the accelerated and trapped electrons can escape from the wave field to produce field-aligned beams which ultimately result in auroral brightening. The electron distributions associated with this process are very similar to those observed by the NASA Polar satellite when large amplitude shear Alfvén waves are present, indicating that electron trapping by shear Alfvén waves could be occurring in the magnetospheric plasma sheet. The self-consistent plasma simulation results reported in this Letter reproduce observable features associated with shear Alfvén waves in natural plasma environments, and provide closure on the link between Alfvén waves and the aurora.

C. E. J. W. is supported by the Canadian Space Agency.

*cwatt@phys.ualberta.ca

[1] S. B. Mende, C. W. Carlson, H. U. Frey, T. J. Immel, and J. C. Gérard, J. Geophys. Res. 108, 8010 (2003).

[2] A. Hasegawa, J. Geophys. Res. 81, 5083 (1976).

[3] C. K. Goertz and R. W. Boswell, J. Geophys. Res. 84, 7239 (1979).

[4] A. Keiling, J. R. Wygant, C. A. Cattell, W. Peria, G. Parks, M. Temerin, F. S. Mozer, C. T. Russell, and C. A. Kletzing, J. Geophys. Res. 107, 1132 (2002).

[5] C. C. Chaston, C. W. Carlson, J. P. McFadden, R. E. Ergun, and R. J. Strangeway, Geophys. Res. Lett. 34, L07101 (2007).

[6] R. L. Lysak and W. Lotko, J. Geophys. Res. 101, 5085 (1996).

[7] C.A. Kletzing, S.R. Bounds, J. Martin-Hiner, W. Gekelman, and C. Mitchell, Phys. Rev. Lett. 90, 035004 (2003).

[8] C.E. J. Watt and R. Rankin, Plasma Phys. Controlled Fusion 50, 074008 (2008).

[9] C. E. J. Watt and R. Rankin, J. Geophys. Res. 112, A04214 (2007).

[10] R. Rankin, C. E. J. Watt, and J. C. Samson, Geophys. Res. Lett. 34, L23103 (2007).

[11] P. Janhunen, A. Olsson, C. T. Russell, and H. Laakso, Space Sci. Rev. 122, 89 (2006).

[12] J. R. Wygant et al., J. Geophys. Res. 107, 1201 (2002).

[13] C. E. J. Watt, R. Rankin, I. J. Rae, and D. M. Wright, J. Geophys. Res. 110, A10S07 (2005). 